\title{
DERECHOS SEXUALES Y REPRODUCTIVOS: CONFIDENCIALIDAD Y VIH/SIDA EN ADOLESCENTES CHILENOS
}

\author{
Ester Valenzuela Rivera y Lidia Casas Becerra*
}

\begin{abstract}
Resumen: Los adolescentes enfrentan obstáculos culturales e institucionales al momento de concurrir a los servicios de salud en busca de atención en salud sexual y reproductiva. Los jóvenes que se autoperciben de riesgo en VIH/SIDA experimentan discriminación por consideraciones socioeconómicas, y se vulnera su derecho a acceder a servicios de salud bajo un marco de confidencialidad, desconociendo el principio de autonomía progresiva y la evolución de sus facultades. La Convención Internacional de los Derechos del Niño (CIDN), ratificada por Chile, provee un marco jurídico que propicia un diálogo y una nueva relación de los adolescentes con la familia, el Estado y la sociedad, e instala un nuevo paradigma a partir del cual la infancia y la adolescencia son consideradas sujetos de derechos.
\end{abstract}

Palabras clave: adolescentes, VIH/SIDA, autonomía progresiva, confidencialidad

\section{REPRODUCTIVE AND SEXUAL RIGHTS: CONFIDENTIALITY AND HIVIAIDS IN CHILEAN ADOLESCENTS}

\begin{abstract}
Adolescents face cultural and institutional obstacles when visiting health care services searching for sexual and reproductive health care. Youngsters who consider themselves at risk for HIV/AIDS undergo discrimination due to socioeconomic reasons, and their right to health care access is infringed under a confidential framework. The principle of progressive autonomy and the evolution of their faculties is ignored. The International Convention for Child's Rights, ratified by Chile, provides a legal framework for dialogue and for a new relationship between adolescents with their family, the State and society, establishing a new paradigm to consider infants and adolescents as subjects to rights.
\end{abstract}

Key words: adolescents, HIV/AIDS, progressive autonomy, confidentiality

\section{DIREITOS SEXUAIS E REPRODUTIVOS: CONFIDENCIALIDADE E HIV/AIDS EM ADOLESCENTES CHILENOS}

Resumo: Os adolescentes enfrentam barreiras culturais e institucionais no momento se utilizarem os serviços de saúde em busca de cuidados de saúde sexual e reprodutiva. Os jovens que se auto percebem como de risco HIV/AIDS, são discriminados por questóes sócio-econômicas e se enfraquece seu direito de acesso aos serviços de saúde sob um marco de confidencialidade, desconhecendo o princípio de autonomia progressiva e a evolução de suas faculdades. A Convençâo Internacional de Direitos da Criança, ratificada pelo Chile, prevê um marco jurídico que possibilita um diálogo e uma nova relação dos adolescentes com a família, o Estado e a Sociedade, e instala um novo paradigma a partir do qual a infância e a adolescência são considerados como sujeitos de direitos.

Palavras-chave: adolescentes, HIV-AIDS, autonomia progressiva, confidencialidade

* Facultad de Derecho, Universidad Diego Portales, Chile Correspondencia: lidia.casas@udp.cl 


\section{Introducción}

Algunas de las cuestiones más tratadas en el ámbito de la salud son la confidencialidad y el consentimiento informado(1-3), particularmente relevantes en la atención en salud sexual y reproductiva de los adolescentes. Si el tratamiento y la detección del VIH/SIDA han provocado discusiones en torno al alcance de dicha salud, resulta aún más relevante discurrir sobre ello cuando los afectados son adolescentes.

La tendencia mundial es el aumento alarmante del VIH/SIDA en la población joven -entre los 15 y los 24 años- e incluso a una edad más temprana(4). Un enfoque integral para la prevención y el tratamiento del VIH/SIDA requiere de un marco ético sobre la confidencialidad para la población afectada o en riesgo. Ello implica aplicarla en la consejería, considerando la autonomía progresiva de los adolescentes en el ejercicio de sus derechos sexuales y reproductivos.

Las prácticas en Chile atentan contra la protección efectiva de los derechos de los adolescentes. La literatura constata la existencia de una serie de barreras culturales e institucionales cuando los jóvenes desean acceder a métodos de prevención de embarazos o de enfermedades de transmisión sexual $(5,6)$. En los servicios de salud públicos, si un adolescente opta por el uso de preservativo como método preventivo de enfermedades de transmisión sexual (ITS) se enfrenta con dificultades para obtenerlos ${ }^{1}$.

\section{Derechos de la infancia y la adolescencia}

Durante el siglo XX la protección de la infancia vivió trascendentales transformaciones. Desde 1959 en adelante, al alero de la Asamblea General de las Naciones Unidas, se va conformando un corpus normativo sobre la protección de los derechos de los niños, quienes eran considerados como objetos de medidas de protección y no sujetos de derechos.

El desenlace de este proceso es la Convención Internacional de Derechos del Niño (CIDN) en 1989, ratificado por Chile en 1990. Pese a que ha generado la

1 Estas apreciaciones surgen de entrevistas a profesionales de servicios de atención primaria de la Región Metropolitana y VI Región y a profesionales de clínicas privadas y con consulta particular de la Región Metropolitana. Fueron además entrevistados los encargados del Programa de Salud de los Adolescentes del MINSAL en el marco de la elaboración del proyecto de tesis sobre el mismo tema. mayor adhesión en la comunidad internacional $(7)^{2}$, su aplicación no ha sido fácil y ha encontrado los mayores escollos para su concreción en las propias legislaciones internas. Chile no escapa a ello.

La Convención dio un giro sobre la concepción de infancia existente: los niños niñas y adolescentes pasan a tener el estatus de sujetos de derechos, con derecho a actuar en favor de su propio bienestar, tomándose en cuenta su participación y, lo que es más importante, entendiendo que dicha participación es fundamental en su desarrollo.

El niño y niña son ahora definidos conforme a sus atributos y derechos respecto de su familia, del Estado y de la sociedad. Ello implica la protección general de sus derechos humanos y una complementaria, traducida en los derechos contenidos en la Convención.

La CIDN define niño/a, señalando que “...es todo ser humano menor de dieciocho ańos de edad, salvo que, en virtud de la ley que le sea aplicable, haya alcanzado antes la mayoría de edad" (Artículo $1^{\circ} \mathrm{CIDN}$ ). No establece un rango de edad que distinga entre niñez y adolescencia; esta última no es un concepto explícito en la Convención. "En realidad el significado de este artículo es que debe protegerse al niño hasta la edad de 18 años como mínimo, pero que como el niño también es un sujeto de derecho debe beneficiarse de cierta autonomía antes de los 18 años. Por esta razón, el artículo $1^{\circ}$ de la Convención queda relativamente abierto" (8).

Aun cuando la Convención explicita el concepto de "adolescencia", no establece la existencia de etapas. El artículo $5^{\circ}$, sin embargo, reconoce una evolución de las facultades desde la infancia a la adolescencia. En otras disposiciones se introducen los términos "edad" y "madurez", manifestadas cuando se consagran los derechos a la libertad de expresión, de pensamiento, conciencia y religión, cuyo ejercicio alude a la evolución de facultades de los niños. Por otra parte, la Convención acepta que los padres den dirección y orientación apropiadas al nińo, pero que, al hacerlo, actúen considerando la evolución de sus facultades, edad y madurez.

2 Hasta el17 de abril de 2007, la CIDN había sido ratificada por 140 Estados, véase http://www.ohchr.org/english/countries/ratification/11. htm. Es la primera Convención más ratificada en la comunidad internacional. 
La Organización Mundial de la Salud (OMS) define la adolescencia como "el período de la vida en el cual el individuo adquiere la capacidad de reproducirse, transita de los patrones psicológicos de la nińez a la adultez y consolida su independencia económica”(9), indicando que los adolescentes serían todos aquellos sujetos entre los 10 y los 19 años de edad(9).

El Comité de los Derechos del Niño, haciendo eco de la definición de la OMS, se refiere a la adolescencia como "... un período caracterizado por rápidos cambios físicos, cognoscitivos y sociales, incluida la madurez sexual y reproductiva, la adquisición gradual de asumir comportamientos y funciones de adultos, que implican nuevas obligaciones y exigen nuevos conocimientos teóricos y prácticos" (10). Los adolescentes se encuentran en una situación de mayor vulnerabilidad por los cambios que enfrentan; su etapa constituye una transición, hay un cambio en las relaciones familiares, búsqueda de identidad personal y gestión de su propia $\operatorname{sexualidad}(10)$.

Para Erikson, "los adolescentes de hoy y mañana están luchando permanentemente por definir nuevos tipos de conducta que sean aplicables a sus vidas; sin embargo, esto está dentro del contexto de dos factores culturales (...) el escepticismo respecto de toda autoridad, la negativa a definir la autoridad auténtica o a rehusar asumir la autoridad que les pertenece por derecho y por necesidad"(11) y la existencia de un hedonismo extraordinario -empleando la palabra en su sentido más amplio-, que implica una desacralización de la vida y la actitud de que toda experiencia es permisible e incluso deseable.

\section{Una etapa de vulnerabilidad}

La adolescencia es una etapa con procesos de socialización en la que los jóvenes están pendientes de la aprobación social y, sobre todo, de la de sus pares; esto debilita su autoimagen y su autoestima haciéndolos vulnerables a las drogas, al alcoholismo, a agresiones, accidentes, etc. (12). La alta prevalencia de comportamientos de riesgo, especialmente entre los 16 y 24 años, podría generar hábitos negativos para la salud por el resto de la vida. Estos comportamientos se traducen no sólo en una mayor probabilidad de desarrollar enfermedades en el futuro, sino en problemas que sufren ya los propios adolescentes, como el embarazo no deseado, las enfermedades de transmisión sexual o los accidentes que encabezan la patología en esta edad.

\section{Derechos sexuales y reproductivos}

Según antecedentes aportados por UNICEF, en América Latina y el Caribe más del 50\% de los jóvenes menores de 17 años han tenido relaciones sexuales. Estudios realizados en Chile -con diversas metodologías- advierten una iniciación sexual más temprana, tanto en hombres como en mujeres: el 60\% de éstas se inician sexualmente entre los 15 y 18 años, mientras que entre los varones el 66\%(13).

Más de la mitad de las infecciones por VIH que se producen en la actualidad afectan a jóvenes entre 15 y 24 años de edad. Según el informe de la UNICEF, "todos los días, aproximadamente 1.700 nińos y niñas contraen VIH. En la actualidad se estima que existen en el mundo aproximadamente 2,1 millones de menores de 15 años que viven con VIH. En 2003, cerca de 630.000 nińos y niñas contrajeron el virus. Agrega que la vía principal de contagio entre adolescentes son las relaciones sexuales sin protección(4). En Chile ha ido en aumento el número de personas contagiadas: la tasa, que en los 80 era de 1,3 por 100 mil habitantes, ascendió a 7,1 en el quinquenio 2000-2005(14).

La salud reproductiva entraña la capacidad de disfrutar de una vida sexual satisfactoria y de procrear sin riesgos; asimismo, la libertad para decidir cuándo, con quién y con qué frecuencia hacerlo(15). Los determinantes de salud, tal como sostienen Correa y Petchetscky, requieren de "condiciones facilitantes" para hacer efectivos el derecho a la salud, la integridad y la autonomía reproductiva(16). Ello significa obtener información y tener acceso a métodos seguros, eficaces, asequibles y aceptables para regular la fecundidad y evitar el contagio con ITS.

La atención en salud debe brindarse de acuerdo con los derechos sexuales y reproductivos, es decir, respetando y garantizando el ejercicio de aquellas libertades y derechos. Aun cuando estos derechos no se incluyen en un solo documento internacional, están en íntima conexión con los consagrados como fundamentales, tales como el derecho a la vida, a la integridad física y psíquica, a la salud, a la intimidad, a la no discriminación, entre otros. Debemos recordar que el derecho internacional es un cuerpo mínimo y subsidiario y que los tratados internacionales seńalan derechos en su contenido esencial primario o básico(17), pudiendo ser detallados o ampliados conforme a la práctica e interpretación. Por ello, los tratados internacionales 
sobre derechos humanos no deben interpretarse como una lista taxativa, sino como espacio abierto a la maximización del sistema total de derechos personales. Así debe entenderse la CIDN.

La Constitución chilena no hace referencia explícita a derechos sexuales; sin embargo, sí se refiere al tema de la autonomía sexual. Ello fue patente en la despenalización de las relaciones sexuales consentidas entre adultos del mismo sexo, derogándose el antiguo artículo 365 del Código Penal(18). Toda la normativa sobre esterilización voluntaria(19) y, posteriormente, el Decreto Supremo del Ministerio de Salud referido a Normas Nacionales sobre Regulación de la Fecundidad, proveen un marco teórico y técnico que recoge los derechos sexuales y reproductivos(20). No obstante, el tratamiento a los adolescentes en salud es aún ambiguo; las normas aluden a ellos como un grupo que requiere atención especial y establecen la necesidad de confidencialidad de la información basada en los principios éticos(20).

Desde octubre de 2000 se encuentra en discusión en el Congreso un proyecto de ley sobre autonomía y control sobre el cuerpo, confidencialidad de la información relacionada con la salud sexual y reproductiva y conveniencia de un acceso a información clara, comprensible y completa sobre métodos de regulación de la fertilidad y de prevención de enfermedades de transmisión sexual y SIDA. Asimismo, el proyecto prohíbe toda forma de discriminación en el ejercicio de los derechos sexuales y reproductivos, estableciendo explícitamente como criterio de sospecha de discriminación la edad y la orientación sexual(21).

La ley también sugiere reconocimiento de la autonomía progresiva. En materia civil, para efectos del Derecho de Familia y patrimoniales, no hay un tratamiento unívoco(22). El adolescente está facultado para un reconocer un hijo sin intervención de la autoridad paterna y puede testar y contraer matrimonio desde los 16 años ${ }^{3}$. También está en condiciones de contratar, ser considerado capaz para efectos de la administración de su peculio profesional y se le otorga la facultad de celebrar un contrato de trabajo con disposiciones que lo protejan.

Por su parte, la tendencia en el Derecho Penal ha sido reconocer la responsabilidad que le cabe al adolescente

3 Artículo 5 Ley de Matrimonio Civil No 19.947 publicada en el Diario Oficial, 17 de mayo de 2004. a partir de los 14 ańos frente a la infracción de la ley ${ }^{4}$. En materia sexual, y reconociendo que el inicio de la actividad sexual sea precoz, la misma ley 19.927 recoge la idea de que los menores no sean objeto de sanción penal bajo ciertas hipótesis.

La noción de autonomía progresiva en conexión con los derechos sexuales y reproductivos ha sido recogida en el Derecho Comparado a partir de las decisiones de los tribunales, asentando el reconocimiento de derechos personalísimos de los adolescentes y las limitaciones a la autoridad parental en el ámbito de la salud sexual y reproductiva.

Es interesante advertir cómo las cuestiones de la autonomía progresiva y el control parental fueron resueltas por la Corte de Apelaciones de Santiago en el caso Zalaquett y otros contra la Ministra de Salud(23). Un alcalde y un par de padres adujeron que las Normas Nacionales de Regulación de la Fecundidad afectaban el derecho de propiedad de los padres sobre la educación de sus hijos, lo que atentaría contra la autoridad de éstos, pues se permitía la entrega de métodos anticonceptivos sin su conocimiento ni su consentimiento(23). La Corte rechazó por unanimidad los fundamentos del recurso, estableciendo que la medida no afecta el ejercicio de este derecho: "Los padres, no obstante el acto cuestionado, siempre podrán educar a sus hijos en las cuestiones de la sexualidad y regulación de la fertilidad de manera que en su concepto les parezca adecuada, incluso expresamente podrán formarlos para que no recurran en circunstancia alguna a la ingesta del anticonceptivo de emergencia, sin que en su afán de contrariar la política gubernamental puede acarrearles consecuencia negativa alguna, porque importa el ejercicio del derecho constitucional de educar a los hijos" $\left(23\right.$, cons. $\left.7^{\circ}\right)$.

Los padres intentan enseñar valores y creencias, pero los hijos están en condiciones, por sí solos, de concretar su propio plan de vida, adoptando, modificando o rechazando aquellos valores o creencias que los padres intentaron inculcar.

\section{VIH/SIDA y adolescentes}

La comunidad internacional se ha preocupado de la situación de la infancia afectada y sus derechos en

4 Ley 20.084 sobre Responsabilidad Penal Juvenil publicada en el Diario Oficial, 7 de diciembre de 2005. 
el contexto del VIH/SIDA. Se han definido algunos principios para reducir la vulnerabilidad de los niños a la infección y para protegerlos de la discriminación por causa de su condición de seropositivo real o pre$\operatorname{sunta}(24)$.

Según estos principios, los Estados deben garantizar el derecho del niño a la vida, a la supervivencia y al desarrollo, evitar la separación de sus padres o familias por esta causa y asegurar que tengan acceso a información sobre el VIH/SIDA y a los medios de prevención, para lo cual deben eliminar los obstáculos sociales, culturales, políticos o religiosos que impiden este acceso, y reconocer el derecho de los nińos a la confidencialidad e intimidad con respecto a su condición de seropositividad(24).

Esto implica el reconocimiento de que las pruebas para la detección del VIH deben ser voluntarias y realizarse con el consentimiento informado de la persona afectada, el que debe obtenerse en el contexto de consejería previa a las pruebas. Si intervienen los tutores legales del niño, deberá prestarse la debida atención a la opinión de éste, si es suficientemente mayor o maduro para opinar al respecto(24).

El Comité de los Derechos del Niño, en su "Observación General (3) El VIH/SIDA y los derechos del nińos", sostiene que la presencia del VIH/SIDA repercutirá en todos los ámbitos y derechos de la vida de un niño, por lo cual insta a los Estados a que sus servicios de salud sean suficientemente receptivos a las necesidades de los menores de 18 años, en particular de los adolescentes(25).

Sobre los servicios y la confidencialidad, el Comité ha puesto especial énfasis en que el nińo debiera tener un mejor acceso a servicios de salud; que éstos sean amigables y le permitan participar en las decisiones que afectan a su salud; que actúen con confidencialidad y no supongan juicios de valor; que no requieran el consentimiento parental ni sean discriminatorios. También, que la condición seropositiva, cuando se trate de un adolescente suficientemente maduro, no sea conocido por terceros, incluyendo a los padres(25, pár. 24).

Para ello se requiere de personal calificado "que respete cabalmente el derecho del nińo a la vida privada y a no sufrir discriminación respecto del acceso a la información sobre el VIH, (que) el asesoramiento y las pruebas de detección se lleven a cabo de manera voluntaria, (que) el nińo tenga conocimiento de su estado serológico con respecto al VIH, (que) tenga acceso a servicios confidenciales de salud reproductiva $\mathrm{y}$, gratuitamente o a bajo costo, a métodos o servicios anticonceptivos" (25 pár. 20 y 11).

\section{Autonomía}

La noción de salud sexual y reproductiva ha sido generalmente comprendida en forma limitada por los encargados de las decisiones en políticas públicas. Este hecho es especialmente problemático cuando los titulares del derecho son personas cuya capacidad para elegir es reducida por la minoría de edad. Históricamente, los nińos y los adolescentes han sido tratados más como objetos que como sujetos de preocupación por su situación de pobreza o irregularidad. Se los ha definido a partir de sus carencias - como la capacidad jurídica- y no tanto en razón de los derechos de los que son titulares(26).

Si bien la CIDN cambia este paradigma, algunos adultos insisten en considerar a los nińos como arcilla moldeable a la luz de sus aspiraciones y exigencias(27), o que la relación adulto-niño descansa sobre la base de amor, cuidado y altruismo, por lo cual el espacio familiar es protegido de intervención(28).

La idea del adolescente como titular de derechos colisiona, con mucha frecuencia, con los deseos de los padres(29). La autonomía es una condición esencial para el ejercicio real de los derechos sexuales y reproductivos. En el caso de los adolescentes, ella es adquirida a través del tiempo, en razón de la evolución de sus facultades, de su edad y madurez.

La CIDN señala que el ejercicio de los derechos del niño será en consonancia con la evolución de sus facultades; esta es la antesala al concepto de evolución progresiva de competencia del niño para ejercer sus derechos con creciente autonomía. El ejercicio de la autoridad, cualquiera sea su naturaleza jurídica, se orienta y limita por los derechos que el ordenamiento jurídico reconoce al niño, considerando, además, los principios de autonomía progresiva en el ejercicio de sus derechos y de participación en todos los asuntos que le afecten $(26, p .3)$.

Señala, además, las funciones parentales de orientación y dirección, y que, en subsidio, ellas recaen en el Estado. Queda claro que el ejercicio de derechos 
es creciente según edad y evolución de facultades y, asimismo, la adquisición de responsabilidades por los actos individuales.

Fijar una edad a partir de la cual consideremos que los adolescentes pueden actuar con autonomía sexual es complejo, y en muchos casos el límite puede ser arbitrario. Sin embargo, lo razonable sería armonizar con la legislación nacional en la que se determinan deberes y responsabilidades a los jóvenes, en lo civil y en lo penal. De esta forma, establecemos una simetría entre la obligación de cumplir deberes y la capacidad para ejercer derechos, ya que si un adolescente es responsable socialmente y tiene deberes en conformidad a sus facultades -esto es, los 14 años para reconocer un hijo-, lo mismo debiera aplicarse respecto del ejercicio de sus derechos, entre éstos los sexuales y reproductivos.

La importancia de incorporar la perspectiva de autonomía progresiva en el análisis de los derechos de la infancia y adolescencia responde a la necesidad de visualizar la vulneración de importantes derechos consagrados en la Convención.

La Observación General número 14 del Comité de Derechos Económicos, Sociales y Culturales(30), cuando desarrolla el derecho a la salud, establece "el deber por parte de los Estados miembros de proporcionar a los adolescentes un entorno seguro y propicio que les permita participar en la toma de decisiones que afecten su salud (...) el ejercicio del derecho a la salud por parte de los adolescentes dependerá de la atención", que ésta sea respetuosa a los jóvenes, tomando en cuenta su derecho a la confidencialidad y la vida privada, y menciona la necesidad de establecer servicios adecuados de salud sexual y reproductiva(30).

La postura de quienes niegan el derecho de los jóvenes a recibir información y servicios sobre la salud reproductiva en confidencialidad suele revelar el temor de que se adelantaría su sexualidad en forma precoz(31). En un sentido complementario, se discute que estas políticas o programas puedan propiciar la ruptura del vínculo entre sexualidad, amor, compromiso y reproducción. Bajo estos supuestos, se suele argumentar que incorporar la presencia de los padres se ampara en el interés superior del niño recogido en la Convención americana. Quienes así arguyen sostienen la visión de que niños y adolescentes siguen siendo objeto de protección y control de parte de los padres u otros adultos. Esto es lo que Cillero denomina "paradoja": la negación de la autonomía para asegurar los derechos de los adultos $(27$, p. 60). Si existe obligación del Estado de fortalecer la misión educativa de la familia, ello se produce en términos de alcanzar la autonomía del adolescente y no de negarla.

La posibilidad del ejercicio de los derechos en forma autónoma -con criterios de progresividad de acuerdo con la edad-implica el abandono de prácticas de subordinación de niños y adolescentes a sus padres, a las instituciones y a los adultos en general, y el reemplazo por funciones de orientación y dirección para que ellos ejerzan los derechos de los cuales son titulares. El desafío es aceptar las diferencias y ampliar los espacios de decisión, de diálogo y de construcción colectiva, en los cuales los adolescentes encuentren su lugar desde sus particularidades y anhelos(32).

Es posible pensar que la idea de "suficiente madurez" -que debe ser considerada por los prestadores y que se erige como guía en la práctica clínica- no hace sino trasladar la potestad parental al control biomédico. Admitiendo esta prevención, la idea permite, al menos, que los adolescentes encuentren espacios de autonomía en el ámbito de la salud cuando ya se han iniciado sexualmente.

\section{Confidencialidad y adolescentes}

La salud no puede ser percibida aisladamente de consideraciones de clase y de género, y la confidencialidad en el acceso a los servicios de salud está cruzada por ambas. La edad profundizaría la inequidad(32). En el ámbito de la salud sexual y reproductiva se advierte discriminación en contra de las adolescentes sólo por ser mujeres y pobres, y las niñas menores de 14 años sexualmente activas pueden ser objeto de interferencias en su vida privada, toda vez que a los profesionales de la salud pública se les instruye notificar de su actividad sexual(33). Por su parte, los varones de la misma edad atendidos en los servicios de salud -públicos o privados- no son objeto de las mismas intromisiones. Nuestras construcciones sociales de la masculinidad revelan que sería inimaginable que la sexualidad "precoz" de un varón se entendiera como problemática cuando acude a un servicio de salud en busca de profiláticos. Tal como sostienen Dehne y Riedner, en ciertas culturas se espera que los varones adolescentes cuenten con cierta experiencia sexual(34). 
Ahora bien, cuando los adolescentes - mujeres y hombres- se autoperciben de riesgo frente al VIH/SIDA y se presentan como tales ante los prestadores de salud, enfrentan mayores prejuicios y barreras al solicitar consejería y servicios para la detección del virus. Ello los priva de su derecho a que la confidencialidad sea resguardada. La tendencia en servicios públicos es que los adolescentes no puedan acceder en reserva, no así cuando son atendidos en servicios de salud privados.

Hay una suerte de vacío legal, pues la Ley sobre VIH 19.779 , en vigencia desde 2001, establece la prohibición de revelar la situación de seropositividad de una persona; sin embargo, la situación del adolescente que concurre solicitando la realización del examen estaría fuera del marco de protección. La ley no previó la situación de los adolescentes, y aún no se dicta el reglamento que debía complementar la ley.

La ambigüedad en el tratamiento de los adolescentes se encuentra reafirmada por lo señalado en el Boletín Técnico No 13 del Consejo Nacional para la Prevención y Control del SIDA (CONASIDA)(35): "Examen VIH en menores de edad: Los menores de edad legalmente "incompetentes" pueden [sic], en ciertos casos, considerarse como competentes para tomar decisiones relacionadas con su salud. En algunos países emerge el concepto de 'mayoría sanitaria' a partir de los 14 años. En Chile no existe consenso ni disposición legal al respecto; sin embargo, en la práctica, las personas menores de edad deben ser adecuadamente informadas de su estado de salud, los procedimientos y los tratamientos que reciben. Lo anterior amerita una evaluación en cada caso dependiendo de la edad del menor; sin embargo, el representante legal o tutor debe ser informado y ser quien decida la realización del test".

La Resolución Exenta № 371, del 2 de febrero de 2001, guarda silencio sobre los adolescentes, por lo cual la única guía es el ya citado Boletín.

Se podría argumentar que no es necesario que exista una norma especial, puesto que el Código Penal sanciona la revelación de secreto ${ }^{5}$. Sin embargo, el secreto se relativiza frente a materias de política sanitaria, y se establece explícitamente la notificación de las ITS conforme con lo establecido en el Código Sanitario, u otras situaciones que se encuentran revestidas de caracteres de delito. De ello no se desprende, empero, que los

5 Artículo 247 del Código Penal. resultados o el acceso al test deban ser comunicados a los miembros de la familia.

Todo lo anterior contrasta con las metas y objetivos declarados por el Poder Ejecutivo en sus planes y programas, tanto en la Política Nacional de la Infancia y Adolescencia como en los Objetivos Sanitarios, ambos para el período 2000-2001. El primero contiene las orientaciones éticas valóricas y operativas y dispone de un instrumento de planificación efectivamente intersectorial (36). Tiene como principios rectores el concepto de nińo como sujeto de derecho especial, el interés superior del niño, la responsabilidad primordial de los padres y la familia en la crianza de sus hijos, la igualdad entre la madre y el padre, y principios orientadores entre los que se encuentran la igualdad de derechos, autonomía progresiva y la no discriminación.

Por su parte, los Objetivos Sanitarios pretenden enfrentar los desafíos derivados del envejecimiento de la población y de los cambios de la sociedad, y disminuir las desigualdades en salud(37). Se refieren también a los cambios de comportamientos de la población adolescente, buscando, a través de la educación sexual integral y el mejoramiento de los servicios, reducir el embarazo en este grupo etario y, con ello, la transmisión de ITS(37).

Sin embargo, ello no se condice con lo que sucede en la práctica: en el plano de la educación sexual, un proceso lento y tortuoso para llevar a cabo un plan nacional; en el de la salud, evidentes barreras de acceso a la consejería y a métodos de regulación de la fecundidad y prevención del VIH/SIDA.

\section{Conclusiones}

La realidad de los servicios públicos de salud en Chile revela que lo que prima es el arbitrio o discreción del profesional para respetar la confidencialidad de los menores de edad en temas relacionados con la salud sexual y reproductiva.

Pese a que el gobierno puede emplear el marco de la CIDN para asegurar el fomento y defensa de los intereses superiores de los nińos respecto del VIH/SIDA, no se advierte una consonancia entre las políticas y las obligaciones, con lo cual la actuación del Estado se queda sólo en declaraciones.

No hay servicios sensibles a las necesidades especiales de los adolescentes y, por el contrario, han dado lugar 
a mayores vulneraciones. Para ser consistentes, una preocupación efectiva implicará transformaciones radicales en la formulación e implementación de políticas públicas. De no materializarse, las obligaciones que Chile ha suscrito quedarán en meros discursos.

\section{Referencias}

1. Cook R, Dickens B. Recognizing adolescents 'evolving capacities' to exercise choice in reproductive health care. International Journal of Gynecology and Obstetrics 2000; 70(1): 13-21.

2. Casas L, Isla P. Confidencialidad de la información médica: Análisis Jurídico de la situación en Chile. En: Casas L, Dides C, Isla P. Confidencialidad de la Información y consentimiento informado en salud sexual y reproductiva. Santiago de Chile: Corporación de Salud y Políticas Sociales; 2002: 91-118.

3. Dides C. El proceso de consentimiento informado en el marco de los derechos sexuales y reproductivos. En: Casas L, Dides C, Isla P. Confidencialidad de la Información y consentimiento informado en salud sexual y reproductiva. Santiago de Chile: Corporación de Salud y Políticas Sociales; 2002: 121-140.

4. Bellamy C. Informe del Estado Mundial de la Infancia 2005. La Infancia Amenazada. Nueva York: 2004: UNICEF. Available from URL: http://www.unicef.cl/centrodoc/pdf/estados/sowc05_sp.pdf

5. Hunt P. Report of the Special Rapporteur. The right to the enjoyment of the highesr attainable standard of physical and mental health. E/CN.4/2004/49 16 of february 2004.

6. Cook R, Dickens B, Fathalla, M. Salud Reproductiva y Derechos Humanos. Integración de la medicina, la ética y el derecho. Bogotá: Oxford y PROFAMILIA; 2003.

7. Schöpp-Schilling B. Reservations to the Convention on the Elimination of All Forms of Discrimination Against Women: An Unresolved Issue or (no) new Developments? In: Ziemele I, ed. Reservations to Human Rights Treaties and the Vienna Convention Regime: Conflict, Harmony and Reconciliation. Boston: Martinus Nijhoff Publishers; 2004: 6.

8. Hodgkin R, Newel P. Manual de Aplicación de la Convención sobre Derechos de los Niños. Ginebra: UNICEF; 2001: 82.

9. OMS. The Health of Young People. Geneva: OMS; 1993.

10. Comité de Derechos del Niño. Observación General No 4. La salud y el desarrollo de los adolescentes en el contexto de la Convención sobre los Derechos del Niño. CRC/GC/2003/4, 21 de julio de 2003.

11. Erikson EH. Sociedad y Adolescencia. México: Andrómeda; 1989: 162.

12. Erickson EH. El Ciclo Vital Complementado. Barcelona: Paidós; 2000: 162.

13. Educación Serie Bicentenario. Comisión de Evaluación y Recomendaciones sobre Educación Sexual. Santiago de Chile: Ministerio de Educación; 2005: 22.

14. CONASIDA. Evolución del VIH/SISA en Chile 1985 a 2005. Santiago: MINSAL; 2006: 7. Disponible en http://epi. minsal.cl/epi/html/bolets/reportes/Pais_86-05\%20vih\%20sida\%20epi.pdf

15. Naciones Unidas. Plataforma de Acción. Conferencia Internacional sobre Población y Desarrollo de El Cairo. Nueva York: UN; 1994.

16. Correa S, Petchesky R. Reproductive and Sexual Rights: A feminist Perspective, Population Policies Reconsidered. In: Sen G, Germain A, Chen L, eds. Health, Empowerment, and Rights. Nueva York: Harvard Center for Population and Development Studies and International Women's Health Coalition; 1994: 109-110.

17. Herrendorf D, Bidart G. Principios de Derechos Humanos y Garantías. Buenos Aires: Editar; 1991.

18. Cabal L, Lemaitre J, Roa M, eds. Cuerpo y Derecho. Legislación y Jurisprudencia en América Latina. Bogotá: Universidad de los Andes, Temis, y Center for Reproductive Rights; 2001.

19. Ministerio de Salud. Resolución No 2326 Exenta que fija directrices para los servicios de salud sobre esterilización femenina y masculina. Santiago de Chile: MINSAL; 30 de noviembre de 2000.

20. Decreto Supremo $N^{\circ} 48$ del Ministerio de Salud que aprueba las Normas Nacionales Sobre Regulación de la Fertilidad, 2007.

21. Proyecto de ley marco sobre derechos sexuales y reproductivos. Boletín No 2608-11.

22. Barcia Lehman R. Sobre la capacidad de los adolescentes para recibir la denominada píldora del día después. Revista de Derecho Privado 2006; 7: 137-158.

23. Corte de Apelaciones. Zalaquett con Ministra de Salud. Rol 4693-06, 10 de noviembre de 2006. 
24. Programa Conjunto de las Naciones Unidas sobre VIH/SIDA. Los Niños y el VIH/SIDA. Documento de Información de ONUSIDA. Actualización del Documento de Información para la campaña mundial contra el SIDA de 1997, febrero de 1999: 4. (Documento en Internet) Disponible en http://data.unaids.org/pub/Report/1999/19990201_children_ es.pdf

25. Comité de Derechos del Niño, Observación General 3. El VIH/SIDA y los derechos del niño. CRC/GC/2003/3, 17 de marzo de 2003.

26. Cillero Bruñol M. Infancia Autonomía y Derechos: Una Cuestión de Principios. En: Derecho a Tener Derecho, Tomo IV. Montevideo: UNICEF-IIN; 1999: 3. (Documento en Internet) Disponible en http://www.inau.gub.uy/Biblioteca/ cillero.pdf

27. Salazar G, Pinto J. Historia Contemporánea de Chile, V. Niñez y Juventud. Santiago de Chile: LOM; 2002: 7.

28. Freeman M. Tomando más en serio los derechos de los nińos. Revista de Derechos del Niño 2006; 3-4: 257-258.

29. Burt R. El tratamiento de menores sin el consentimiento de los padres. In: Beloff M, comp. Derecho, infancia y familia. Barcelona: Gedisa; 2000: 189-190.

30. Comité de Derechos Económicos, Sociales y Culturales. Observación General número 14 sobre el Derecho a la Salud. E/C.12/2000/4, CESCR, Observación General 14, 15 de abril de 2000: 23.

31. Libertad y Desarrollo. Píldora del Día Después y Embarazo Adolescente. Temas Públicos 2006; 788: 2. (Sitio en Internet) Disponible en http://www.lyd.org

32. Palma I. Salud y Derechos Sexuales y reproductivos de adolescentes y jóvenes en el contexto de la reforma de salud en Chile. 2001. (Sitio en Internet) Disponible en http://www.paho.org/English/DPM/GPP/GH/adolescents.pdf

33. Casas L. Confidencialidad de la información médica, el derecho a la salud y el consentimiento sexual de los adolescentes. Revista de la Sociedad Chilena de Obstetricia y Ginecología Infantil y de la Adolescencia 2005; 12(3): 94-111.

34. Dehne K, Riedner G. Adolescent. A dynamic concept. Reproductive Health Matters 2001; 9: 17.

35. CONASIDA. Voluntariedad y Consejería en el Examen de VIH. Boletín Técnico No 13, 2000. (Sitio en Internet) Disponible en http://www.conasida.cl/bol/bol13/bol13tec.htm

36. Gobierno de Chile. Politica Nacional a favor de la Infancia y Adolescencia. Santiago de Chile: MIDEPLANP; 2000.

37. Gobierno de Chile. Objetivos Sanitarios 2000-2010. Resumen Ejecutivo. El Vigía. Boletín de Vigilancia en Salud Pública 2002; 5: 15. (Documento en Internet) Disponible en http://epi.minsal.cl/epi/html/elvigia/vigia15.pdf

Recibido: 6 de julio de 2007.

Aceptado: 27 de julio de 2007. 